\title{
A Complex Analytic Study on the Theory of Fourier Series on Compact Lie Groups
}

By

\author{
Yutaka SABURI*
}

\begin{abstract}
The characterization of Fourier series of real analytic functions and hyperfunctions on compact Lie groups is discussed by means of complex analysis.
\end{abstract}

\section{\$0. Introduction}

The aim of the present work is to give the characterization of Fourier series of real analytic functions and hyperfunctions on compact Lie groups by means of complex analysis.

Here let us review the history of the studies concerning this subject. The concept of hyperfunctions was first introduced by Sato [Sa] in 1958. In [Sa], among other results, he already got the characterization of Fourier series of real analytic functions and hyperfunctions on the one dimensional sphere $S^{1}$. The way he used was complex analysis, more precisely Cauchy's integral theorem. In 1970, Sugiura [Su] gave the characterization of Fourier series of $C^{\infty}$ functions on compact Lie groups. His method was based on the fact that, on a compact Lie group, the Casimir operator becomes an elliptic differential operator and it acts as a scalar operator on each irreducible unitary representation space of the group. In 1973, Hashizume, Minemura and Okamoto [HMO] characterized the Fourier coefficients of real analytic functions and hyperfunctions on real analytic compact Riemannian manifolds with respect to the Laplace-Beltrami operators. Their argument mainly depended on the estimate for the growth of eigenvalues of the Laplace-Beltrami operator obtained in [Mi]. In 1979-80, Morimoto [Mo1, Mo2] discussed on the characterization of the Fourier coefficients of the analytic functionals on the complex sphere or the Lie sphere with respect to spherical harmonics. His work owed mainly to the classical

Received August 15, 1996.

1991 Math. Subject Classification: 22E30, 46F 15.

* Chiba Jr. College. Ichikawa 272-8512. Japan.

Research Supported by Grant-in-Aid for Academic Research, Chiba Jr. College. 
theory of spherical harmonics and the study of the Shilov boundaries of E. Cartan's classical domains of type IV in [Hu]. In 1991, Oshima, Saburi and Wakayama [OSW2] treated the characterization of Fourier coefficients of real analytic functions and hyperfunctions on the so called Martin boundaries of Riemannian symmetric spaces with noncompact linear reductive groups as their isometry groups (cf. also [OSW1]). Their method was similar to that in [Su]. In 1995, by the similar argument used in [HMO], Morimoto and Fujita [FM] got the characterization in the case of the spaces of functions of Gevrey classes and their dual spaces on real analytic compact Riemannian manifolds.

In this paper we shail give a different proof for the result in [HMO] in the case of the compact Lie groups. Our argument is complex analytic. Precisely, we present the matrix elements of irreducible unitary representations of compact groups in the integral form owing to the Borel-Weil theorem. Then we can get a good estimate of the Fourier coefficients by shifting the cycle of the integrals owing to Cauchy's integral theorem. However we note that the method in $[\mathrm{Su}]$ is valid even in our case.

At the end of this introduction we have to add that this work is inspired not only by the works started above, but also by [B1, B2]. In [B1, B2], van den Ban studied on asymptotic behavior of Eisenstein integrals associated with the minimal parabolic subgroup of a connected real semisimple Lie group with finite center. To get a good estimate for the integrals, the method used in those preceding works [A, HC1, HC2, HC3, HC4, E, TV], etc. were mainly based on the study of differential equations satisfied by these integrals. In contrast with those preceding works, he made a good deformation of the cycle of the integrals owing to the Stokes formula. That gave us the quite enough motivation of the shift of the cycle in our problem.

\section{§1. Compllexifieation of a Compact Lie Grourp}

Let $U$ be a connected compact Lie group. It is known that there exists a connected algebraic subgroup $G$ of a complex general linear group $G L(n, \mathbb{C})$ with the following properties. (a) It is stable under conjugate transpose. (b) There exists an isomorphism of $U$ onto $G \cap \mathrm{U}(n)$, where $\mathrm{U}(n)$ is the unitary group. (c) The Lie algebra $g$ of $G$ coincides with the complexification $\mathfrak{u}_{\mathbb{C}}=\mathfrak{u} \oplus$ $\sqrt{-1} \mathfrak{u}$ of the Lie algebra $\mathfrak{u}$ of $G \cap \mathrm{U}(n)$. We identify $U$ with $G \cap \mathrm{U}(n)$, and introduce into $U$ a real analytic structure via this identification. This means a function on an open subset of $U$ is real analytic if and only if it extends to a holomorphic function on an open subset of $G$. The group $G$ is called the Chevalley complexification of $U$. Note that the group $G$ is not only linear algebraic but also real reductive. We denote by $1_{G}$ the identity element of $G$.

Let $\theta$ denote a Cartan involution of $G$ defined as $\theta g=\left(g^{*}\right)^{-1}$ for $g \in G$, where $g^{*}$ denotes the conjugate transpose of $g$. We also denote its differential by 
$\theta$, that is, $\theta X=-X^{*}$ for $X \in \mathrm{g}$. Then the direct sum decomposition $\mathrm{g}=\mathfrak{u} \oplus \sqrt{-1} \mathfrak{u}$ means the Cartan decomposition for $\theta$. Namely $\mathfrak{u}$ is the 1 eigenspace and $\sqrt{-1} \mathfrak{u}$ is the -1 eigenspace for $\theta$, respectively. We denote by exp the exponential mapping of $g$ into $G$. Then we have a Cartan decomposition of $G$. Namely the mapping

$$
U \times \sqrt{-1} u \ni(u, X) \mapsto u \exp X \in G
$$

is a real analytic diffeomorphism. Hence for every $g \in G$ there exist unique elements $u^{c}(g) \in U$ and $X(g) \in \sqrt{-1} \mathfrak{u}$ so that

$$
g=u^{c}(g) \exp X(g) .
$$

Let $\mathfrak{t}$ be a maximal abelian subalgebra of $\mathfrak{u}$, and put $\mathfrak{a}=\sqrt{-1} \mathfrak{t}$ and $\mathfrak{c}=\mathfrak{t}_{\mathbb{C}}=\mathfrak{t}$ $\oplus$ a. Then $a$ is a maximal abelian subspace of $\sqrt{-1} \mathfrak{u}$ and $\mathfrak{c}$ is a Cartan subalgebra of $\mathfrak{g}$. Put $T=\exp \mathfrak{t}, A=\exp \mathfrak{a}$ and $C=\exp \mathfrak{c}=T A$. Then $T$ is a maximal torus of $U$, and $C$ is a Cartan subgroup of $G$. We have another Cartan decomposition of $G$ :

$$
G=U A U
$$

So every $g \in G$ decomposes into the form

$$
g=u_{1}^{C} a^{C} u_{2}^{C}
$$

with $u_{1}^{c}, u_{2}^{C} \in U$ and $a^{C} \in A$. Let $\mathfrak{a}^{\prime}$ and $\mathfrak{c}^{\prime}$ denote the dual spaces of $\mathfrak{a}$ and $\mathfrak{c}$, respectively. Since $\mathfrak{c}=\mathfrak{a}_{\mathbf{C}}$, any element in $\mathfrak{a}^{\prime}$ has a unique natural extension as an element in $\mathfrak{c}^{\prime}$. So we regard the elements in $\mathfrak{a}^{\prime}$ as elements in $\mathfrak{c}^{\prime}$ which are real valued on $\mathfrak{a}$. Let $\Sigma(\mathfrak{g}, \mathfrak{a})$ denote the set of all nonzero restricted roots of $\mathfrak{g}$ with respect to $a$, and $\mathrm{g}^{\alpha}$ the root space for $\alpha \in \Sigma(\mathfrak{g}, \mathfrak{a})$. Note that $\Sigma(\mathrm{g}, \mathfrak{a})$ coincides with the set of all nonzero roots of $\mathfrak{g}$ with respect to $\mathfrak{c}$ in the above sense and that each of the root spaces $g^{\alpha}$ is a complex one dimensional vector subspace. Introducing a lexicographic ordering into $a^{\prime}$ with respect to some basis of $a$, let us denote by $\Psi$ the positive system of $\Sigma(\mathfrak{g}, \mathfrak{a})$ and by $\Delta$ the set of all simple elements in $\Psi$. Put $\mathfrak{n}=\bigoplus_{\alpha \in \Psi} \mathfrak{g}^{\alpha}, \mathfrak{n}^{-}=\theta \mathfrak{n}=\bigoplus_{\alpha \in \Psi} \mathfrak{g}^{-\alpha}, N=\exp \mathfrak{n}$ and $N^{-}=\exp \mathfrak{n}^{-}$. Then $N$ and $N^{-}$are complex nilpotent Lie subgroups of $G$.

Now we have an Iwasawa decomposition of $G$. Namely the mapping

$$
U \times A \times N^{-} \ni\left(u, a, n^{-}\right) \mapsto u a n^{-} \in G
$$

is a surjective real analytic diffeomorphism. Hence for all $g \in G$, there exist unique elements $u(g) \in U, a(g) \in A$ and $\nu^{i,-}(g) \in N^{-}$such that

$$
g=u(g) a(g) \nu^{\imath,-}(g)
$$

and the mapping $g \mapsto\left(u(g), a(g), \nu^{\imath,-}(g)\right)$ of $G$ onto $U \times A \times N^{-}$is real analytic. Since the restriction of the exponential mapping of $g$ to $a$ is injective, writing its 
inverse by $\log _{A}$ we put $H(g)=\log _{A}(a(g))$ for $g \in G$.

Put $B=C N^{-}=T A N^{-}$. Then $B$ is not only a Borel subgroup of the linear algebraic group $G$ but also a minimal parabolic subgroup of the real reductive group $G$. Since $G$ is connected, its open dense subset $N B$ admits the so called Gelfand-Naimark decomposition. Namely the mapping

$$
N \times B \ni(n, b) \mapsto n b \in N B
$$

is a surjective holomorphic diffeomorphism. We note that any element $b$ in $B$ uniquely decomposes into a product $c n^{-}$of elements $c \in C$ and $n^{-} \in N^{-}$because of the fact $C \cap N=\left\{1_{G}\right\}$. We also note that the groups $N, C$ and $N^{-}$are complex Lie subgroups of $G$. Then we see that for all elements $x \in N B$ there exist unique elements $\nu(x) \in N, c(x) \in C$ and $\nu^{-}(x) \in N^{-}$such that

$$
x=\nu(x) c(x) \nu^{-}(x),
$$

and the mapping $x \mapsto\left(\nu(x), c(x), \nu^{-}(x)\right)$ of $N B$ onto $N \times C \times N^{-}$is holomorphic.

We have a relation between the Iwasawa decomposition (1.6) and the Gelfand-Naimark decomposition (1.8):

Lemma 1. Let $n \in N$, then $u(n) \in N B$ and the following holds

$$
\begin{aligned}
& \nu(u(n))=n, \quad c(u(n))=a(n)^{-1} \quad \text { and } \\
& \nu^{-}(u(n))=a(n) \nu^{i,-}(n)^{-1} a(n)^{-1} .
\end{aligned}
$$

Proof. Let $n \in N$. Then by (1.6) we have

$$
\begin{aligned}
u(n) & =n \nu^{1,-}(n)^{-1} a(n)^{-1} \\
& =n a(n)^{-1}\left(a(n) \nu^{2,-}(n) a(n)^{-1}\right) .
\end{aligned}
$$

Since $C$ normalizes $N^{-}$, this equality shows $u(n) \in N B$. Hence we have (1.9) by the uniqueness of the decomposition (1.8).

\section{§2. $\quad$ Hrreducible Unitary Representations of $\mathbb{U}$}

In this section we review a summary on the irreducible unitary representations of the compact connected Lie group $U$ (cf. [W, $\S 1.7$ in Chap. 1] and [Kn, $\S 7$ in Chap. V]).

Put $B(X, Y)=\operatorname{Tr}(X Y)$ for $X . Y \in \mathrm{g}$. Then $B$ gives an invariant symmetric bilinear form on $g$. Since the elements in $\sqrt{-1} \mathfrak{u}$ are Hermitian symmetric matrices, the restriction of $B$ to $\sqrt{-1} \mathfrak{u}$ is real valued and positive definite. Hence we see that both $B$ and its restriction to $c$ are non-degenerate and that for any $\mu \in \mathfrak{c}^{\prime}$ there exists unique $H_{\mu} \in \mathfrak{c}$ such that $\mu(H)=B\left(H, H_{\mu}\right)$ holds for all $H \in \mathfrak{c}$. We can then define a symmetric non-degenerate bilinear form on $\mathfrak{c}^{\prime}$ by putting $(\sigma, \tau)=B\left(H_{\sigma}, H_{\tau}\right)$ for $\sigma, \tau \in \mathfrak{c}^{\prime}$. Since the restriction of this bilinear form to $\mathfrak{a}^{\prime}$ is positive definite, it gives an inner product on $\mathfrak{a}^{\prime}$. An element $\lambda$ in $\mathfrak{c}^{\prime}$ is 
said to be dominant if it satisfies the condition $(\lambda, \alpha)>0$ for all $\alpha \in \Psi$.

Put $L=\left\{H \in \mathrm{t}\right.$; $\left.\exp H=1_{G}\right\}$. An element $\mu$ in $\mathfrak{c}^{\prime}$ is said to be analytically integral or $T$-integral if it satisfies the condition $\mu(L) \subset 2 \pi \sqrt{-1} \mathbb{L}$. Since $L$ spans the whole space $t$ over $\mathbb{R}$, we see that $T$-integral elements are in $\boldsymbol{a}^{\prime}$. We note that every element in $\Sigma(\mathfrak{g}, \mathfrak{a})$ is $T$-integral (cf. [He, Lemma 6.5 in Chap. VII]). We put

$$
\Lambda=\left\{\lambda \in c^{\prime} ; \lambda \text { is dominant and } T \text {-integral }\right\} .
$$

As for the algebraic structure of the irreducible unitary representations of the compact connected Lie group $U$, the followings are well-known. (A) Every irreducible unitary representation of $U$ is finite dimensional and its differential gives rise to a highest weight module of $U(\mathrm{~g})$, the universal envelopping algebra of g. (B) For any $\lambda \in \Lambda$, there exists an irreducible unitary representation $\Phi_{\lambda}$ of $U$ with the highest weight $\lambda$. (C) For any distinct $\lambda$ and $\mu$ in $\Lambda, \Phi_{\lambda}$ and $\Phi_{\mu}$ are not equivalent. (D) The set $\left\{\Phi_{\lambda}\right\}_{\lambda \in \Lambda}$ gives a complete representative system of the equivalent classes of irreducible unitary representations of $U$. (E) For $\lambda \in$ $\Lambda$, every weight $\sigma$ of $\Phi_{\lambda}$ is of the form $\sigma=\lambda-\sum_{\alpha \in \Delta} m_{\alpha} \alpha\left(m_{\alpha} \in \mathbb{Z}_{\geq 0}\right)$, hence is $T$-integral. Furthermore it satisfies the following estimate:

$$
\|\sigma\| \leq\|\lambda\|
$$

Next, we mention the realization of irreducible unitary representations of $U$ with the given highest weight $\lambda \in \Lambda$, the Borel-Weil theorem. Let $\lambda \in \Lambda$, and put $c^{\lambda}=\exp (\lambda(H))$ for $c=\exp H \quad(H \in \mathfrak{c})$. Then $c^{\lambda}$ defines a single valued holomorphic function on $C$. We put also $\xi_{\lambda}\left(c n^{-}\right)=c^{\lambda}$ for $c \in C$ and $n^{-} \in N^{-}$, then $\xi_{\lambda}$ gives a holomorphic one dimensional representation of $B$. Furthermore we put $\Phi(\lambda)=\operatorname{ind}_{B}^{G} \xi_{\lambda}$, the induced representation of $G$ by $\xi_{\lambda}$. That is, the left regular representation: $\Phi(\lambda ; g) f(x)=f\left(g^{-1} x\right)(g, x \in G)$ of $G$ on a function space

$$
\Gamma(\lambda)=\left\{f \in \mathscr{O}(G) ; f(g b)=\xi_{\lambda}(b)^{-1} f(g) \text { for all } g \in G \text { and all } b \in B\right\},
$$

where $\mathscr{O}(G)$ denotes the space of all holomorphic functions on $G$. Then $\Phi(\lambda)$ is a holomorphic irrenducible representation of $G$ with the highest weight $\lambda$. Introducing the inner product of $L^{2}(U)$ into $\Gamma(\lambda)$, one can make it a Hilbert space. Then the restriction of $\Phi(\lambda)$ to $U$ gives the irreducible unitary representation of $U$ with the highest weight $\lambda$.

For the later use, we give a formula for the $L^{2}(U)$-norm of the functions in $\Gamma(\lambda)$ with $\lambda \in \Lambda$. Put $\rho=2^{-1} \sum_{\alpha \in \Psi}\left(\operatorname{dim}_{\mathbf{R}} g^{\alpha}\right) \alpha=\sum_{\alpha \in \Psi} \alpha$. Then the invariant integral on $U$ is given as

$$
\int_{U} f(u) d u=\int_{N} \int_{T} f(u(n) t) a(n)^{2 \rho} d t d n
$$

for continuous functions $f$ on $U$, where $d n$ is the left invariant measure on $N$ 
normalized so that $\int_{N} a(n)^{2 \rho} d n=1$ and $d t$ the Lebesgue measure on $T$ normalized so that $\int_{T} d t=1$. Let $\lambda \in \Lambda$ and $f \in \Gamma(\lambda)$. Then by Lemma 1 and (2.3) we have

$$
f(u(n) t)=f(n)\left(a(n) t^{-1}\right)^{\lambda}
$$

for all $n \in N$ and all $t \in T$. Since $\Lambda \subset \mathfrak{a}^{\prime}$, we also see that $\left|t^{\lambda}\right|=1$ for all $t \in T$. Therefore we have the following formula for the functions $f$ in $\Gamma(\lambda)$ :

$$
\begin{aligned}
\|f\|^{2} & =\int_{N} \int_{T}|f(u(n) t)|^{2} a(n)^{2 \rho} d t d n \\
& =\int_{N}|f(n)|^{2} a(n)^{2(\lambda+\rho)} d n .
\end{aligned}
$$

We finish this section by recalling a well-known estimate for the matrix elements of $\Phi(\lambda)$.

The restriction of the invatrinat symmetric bilinear form $B$ on $g$ to $\sqrt{-1} u$ gives its inner product: $\langle X, Y\rangle=B(X, Y)$ for $X, Y \in \sqrt{-1} \mathfrak{u}$. Using this inner product and the Cartan decomposition (1.2), we put $\|g\|=\|X(g)\|$ for $g \in G$. Then the followings hold

$$
\begin{array}{ll}
\left\|u g u^{\prime}\right\|=\|g\| & \text { for } u, u^{\prime} \in U \text { and } g \in G, \\
\left\|g^{-1}\right\|=\|g\| & \text { for } g \in G .
\end{array}
$$

For $\lambda \in \Lambda$ we put $d(\lambda)=\operatorname{dim}_{\mathrm{C}} \Gamma(\lambda)$. Since $\Gamma(\lambda)$ is decomposed as the orthogonal direct sum of its weight spaces, we can choose an orthonormal weight basis $\left\{\varphi_{i}(\lambda)=\varphi_{i}(\lambda ; g)\right\}_{1 \leq_{i, j} \leq_{d}(\lambda)}$, and in what follows we will fix it for simplicity. We identify $\Phi(\lambda)$ with its matrix representation $\left[\Phi_{i j}(\lambda)=\right.$ $\left.\Phi_{i j}(\lambda ; g)\right]_{1 \leq i, j \leq d(\lambda)}$, where

$$
\Phi_{i j}(\lambda ; g)=\left(\Phi(\lambda ; g) \varphi_{j}(\lambda), \varphi_{\imath}(\lambda)\right) \quad(g \in G, 1 \leq i, j \leq d(\lambda)) .
$$

Then the following estimate holds:

$$
\left|\Phi_{i j}(\lambda ; g)\right| \leq e^{\|\lambda\|\|g\|} \quad(g \in G, 1 \leq i, j \leq d(\lambda)) .
$$

This is an immediate consequence of the following inequality

$$
\left|\Phi_{i j}(\lambda ; g)\right| \leq\|\Phi(\lambda ; g)\|\left\|\varphi_{j}\right\|\left\|\varphi_{\imath}\right\|=\|\Phi(\lambda ; g)\|
$$

and the decomposition $(1,4)$ and the estimate $(2,2)$.

\section{§3. Fourier Series of Real Analytic Functions on $U$}

The family $\{\Phi(\lambda)\}_{\lambda \in \Lambda}$ gives a complete representative system of the equivalent classes of irreducible unitary representations of $U$. Hence, from the Peter-Weyl theorem, we see that the set 


$$
\left\{d(\lambda)^{1 / 2} \Phi_{i j}(\lambda) ; \lambda \in \Lambda, 1 \leq i, j \leq d(\lambda)\right\}
$$

gives an orthonormal basis of $L^{2}(U)$. Then any function $f$ in $L^{2}(U)$ has an expansion

$$
f=\sum_{\lambda \in \Lambda} d(\lambda) \sum_{1 \leq i, j \leq d(\lambda)}\left(f, \Phi_{i j}(\lambda)\right) \Phi_{i j}(\lambda)
$$

the Fourier series of $f$. For $f \in L^{2}(U)$ and $\lambda \in \Lambda$, we put $\mathscr{F} f_{i j}(\lambda)=\left(f, \Phi_{i j}(\lambda)\right)$ $(1 \leq i, j \leq d(\lambda))$ and $\mathscr{F}_{f} f(\lambda)=\left[\mathscr{F}_{i j}(\lambda)\right]_{1 \leq_{i, j} \leq_{d}(\lambda)}$. For $k \in \mathbb{Z}_{>0}$ let $M(k, \mathbb{C})$ denote the set of all $k-b y-k$ complex matrices, and we put $S(\Lambda)=\prod_{\lambda \in \Lambda} M(d(\lambda), \mathbb{C})$. For $s \in S(\Lambda)$ and $\lambda \in \Lambda$, let us denote the $\lambda$-th component of $s$ by $s(\lambda) \in$ $M(d(\lambda), \mathbb{C})$. Then the space

$$
S^{2}(\Lambda)=\left\{s \in S(\Lambda) ;\|s\|^{2}=\left[\sum_{\lambda \in \Lambda} d(\lambda) \operatorname{Tr}(s(\lambda) s(\lambda) *)\right]^{1 / 2}<\infty\right\}
$$

is a Hilbert space with the Hermitian inner product

$$
\left(s, s^{\prime}\right)=\sum_{\lambda \in \Lambda} d(\lambda) \operatorname{Tr}\left(s(\lambda) s^{\prime}(\lambda) *\right) \quad \text { for } s, s^{\prime} \in S^{2}(\Lambda) .
$$

Again from the Peter-Weyl theorem we see that the mapping $\mathscr{F}: f \mapsto \mathscr{F} f=(\mathscr{F} f$ $(\lambda))_{\lambda \in \Lambda}$ is a unitary operator of $L^{2}(U)$ onto $S^{2}(\Lambda)$. We call $\mathscr{F}$ the Fourier transformation of $L^{2}(U)$. The inverse $\mathscr{F}^{-1}$ of the maping $\mathscr{F}$ is given in the form

$$
\mathscr{F}^{-1} s=\sum_{\lambda \in \Lambda} d(\lambda) \operatorname{Tr}\left(s(\lambda)^{t} \Phi(\lambda)\right) \quad \text { for } s \in S^{2}(\Lambda) .
$$

Now we are going to discuss on the restriction of the Fourier transformation $\mathscr{F}$ of $L^{2}(U)$ to the subspace $\mathscr{A}(U)$ of all real analytic functions on $U$.

Let $V$ be an open subset of $G$. We put

$$
\mathscr{O}_{b}(V)=\left\{f \in C(C l(V)) ;\left.f\right|_{V} \in \mathscr{O}(V)\right\},
$$

where $C l(V)$ denotes the closure of $V$ in $G, C(C L(V))$ the space of all continuous functions on $\mathrm{Cl}(V)$, and $\mathscr{O}(V)$ the space of all holomorphic functions on $V$. Then $\mathscr{O}_{b}(V)$ is a Banach space with the norm $\|f\|=\sup _{g \in C l(V)}|f(g)|$ for $f \in$ $\mathscr{O}_{b}(V)$. For $\varepsilon>0$, we put $V_{\varepsilon}=\{g \in G ;\|g\|<\varepsilon\}$. Then $\left\{V_{\varepsilon}\right\}_{\varepsilon>0}$ consitutes a fundamental neighborhood system of $U$ in $G$. For $0<\varepsilon<\varepsilon^{\prime}$, the natural restriction mapping $\mathscr{O}_{b}\left(V_{\varepsilon^{\prime}}\right) \rightarrow \mathscr{O}_{b}\left(V_{\varepsilon}\right)$ is injective and compact. Hence $\mathscr{A}(U)$ becomes a DFS space with the following inductive limit topology (cf. [Ko]):

$$
\mathscr{A}(U)=\lim \operatorname{ind}_{\varepsilon>0} \mathscr{O}_{b}\left(V_{\varepsilon}\right)=\lim \operatorname{ind}_{k \in \mathbf{Z}_{>0}} \mathscr{O}_{b}\left(V_{1 / k}\right) .
$$

For $\eta \in \mathbb{R}$ we put 


$$
S_{\eta}(\Lambda)=\left\{s \in S(\Lambda) ;\|s\|_{\eta}=\sup _{\lambda \in \Lambda} e^{-\eta|| \lambda i \mid}\left\{\operatorname{Tr}(s(\lambda) s(\lambda))^{*}\right\}^{1 / 2}<\infty\right\} .
$$

Then $S_{\eta}(\Lambda)$ is a Banach space. We also put $S_{\mathscr{A}}(\Lambda)=\cup_{\varepsilon>0} S_{-\varepsilon}(\Lambda)$. For $0<\varepsilon<\varepsilon^{\prime}$, the natural inclusion mapping $S_{-\varepsilon^{\prime}}(\Lambda) \hookrightarrow S_{-\varepsilon}(\Lambda)$ is compact. Hence $S_{\mathscr{A}}(\Lambda)$ is also a DFS space with the following inductive limit topology:

$$
S_{\mathscr{A}}(\Lambda)=\lim \operatorname{ind}_{\varepsilon>0} S_{-\varepsilon}(\Lambda)=\lim \operatorname{ind}_{k \in \mathbf{Z}_{>0}} S_{-1 / k}(\Lambda) .
$$

Now we can state our first assertion.

Theorem 2. (i) The Fourier transformation F gives a topological linear isomorphism of $\mathscr{A}(U)$ onto $S_{\mathscr{A}}(\Lambda)$.

(ii) For $s \in S_{\mathscr{A}}(\Lambda)$ the Fourier series (3.5) converges in the topology of $\mathscr{A}(U)$.

For the proof of this theorem we prepare the following lemma.

Lemma 3. Let $\varepsilon>0$. Then we have

$$
\left|\mathscr{F}_{i j}(\lambda)\right| \leq\|f\|_{\varepsilon} e^{-\varepsilon\|\lambda\|} \quad(1 \leq i, j \leq d(\lambda))
$$

for all $\lambda \in \Lambda$ and all $f \in \mathscr{O}_{b}\left(V_{\varepsilon}\right)$.

Proof. When $\lambda=0, \Phi(\lambda)$ is trivial. Hence (3.10) is obvious in that case. So we may assume that $\lambda \in \mathbb{\Lambda} \backslash\{0\}$. Let $f \in \mathscr{O}_{b}\left(V_{\varepsilon}\right)$. Since $\left.\Phi(\lambda)\right|_{U}$ is unitary, $\Phi\left(\lambda ; u^{-1}\right)=\Phi(\lambda ; u) *$ holds for all $u \in U$, and thus, it follows from (2.4) and (2.5) that

$$
\begin{aligned}
& \mathscr{F}_{i j}(\lambda)=\int_{U} f(u) \Phi_{j i}\left(\lambda ; u^{-1}\right) d u \\
= & \int_{U} f(u) \int_{U} \varphi_{\imath}(\lambda ; u v) \overline{\varphi_{j}(\lambda ; v)} d v d u \\
= & \int_{U^{2}} f\left(u v^{-1}\right) \varphi_{i}(\lambda ; u) \overline{\varphi_{j}(\lambda ; v)} d u d v \\
= & \int_{(N \times T)^{2}} f\left(u\left(n_{1}\right) t_{1} t_{2}{ }^{-1} u\left(n_{2}\right)^{-1}\right) \varphi_{i}\left(\lambda ; n_{1}\right) \overline{\varphi_{j}\left(\lambda ; n_{2}\right)} \\
& \cdot\left(a\left(n_{1}\right) t_{1}^{-1} a\left(n_{2}\right) t_{2}\right)^{\lambda}\left(a\left(n_{1}\right) a\left(n_{2}\right)\right)^{2 \rho} d t_{1} d t_{2} d n_{1} d n_{2} .
\end{aligned}
$$

Here we note that the function $f\left(u\left(n_{1}\right) c_{1} c_{2}{ }^{-1} u\left(n_{2}\right)^{-1}\right)\left(c_{1}{ }^{-1} c_{2}\right)^{\lambda}$ is holomorphic in the variables $\left(c_{1}, c_{2}\right)$ on some neighborhood of $T^{2}$ in $C^{2}$. So, to obtain a good estimate of the integral (3.11) we are going to shift the component $T^{2}$ in its domain of integration. For $\eta \in \mathbb{R}$, put $a(\lambda, \eta)=\exp \left(\eta H_{\lambda} /\|\lambda\|\right) \in A$. Then we see

$$
\left|(a(\lambda ; \eta) t)^{\lambda}\right|=e^{\eta\|\lambda\|} \text { and }\left|(a(\lambda ; \eta) t)^{-\lambda}\right|=e^{-\eta\|\lambda\|}
$$

for all $t \in T$. On the other hand, from (2.7) we see that

$$
\left\{u_{1} c_{1} c_{2}^{-1} u_{2} ; u_{1}, u_{2} \in U, c_{1} \in a(\lambda ; \eta) T \text { and } c_{2} \in a\left(\lambda ;-\eta^{\prime}\right) T\right\} \subset V_{\varepsilon}
$$


for all $\eta$ and $\eta^{\prime}$ with $0 \leq \eta, \eta^{\prime}<\varepsilon / 2$. Hence we can shift the component $T^{2}$ in the domain of integration (3.11) into $a(\lambda ; \varepsilon / 2) T \times a(\lambda ;-\varepsilon / 2) T$ without change of its value. Then by the Schwartz inequality and the formula (2.6), we have

$$
\begin{aligned}
& \left|\mathscr{F}_{i j}(\lambda)\right| \\
\leq & \|f\|_{\varepsilon} e^{-\varepsilon\|\lambda\|} \int_{N^{2}}\left|\varphi_{i}\left(\lambda ; n_{1}\right) \varphi_{j}\left(\lambda ; n_{2}\right)\right|\left(a\left(n_{1}\right) a\left(n_{2}\right)\right)^{\lambda+2 \rho} d n_{1} d n_{2} \\
\leq & \|f\|_{\varepsilon} e^{-\varepsilon\|\lambda\|}\left\|\varphi_{\imath}\right\|\left\|\varphi_{j}\right\|\left[\int_{N^{2}}\left(a\left(n_{1}\right) a\left(n_{2}\right)\right)^{2 \rho} d n_{1} d n_{1}\right]^{1 / 2} \\
= & \|f\|_{\varepsilon} e^{-\varepsilon\|\lambda\|} .
\end{aligned}
$$

Proof of Theorem 2. We are going to prove (i) and (ii) simultaneously. The Fourier transformation $\mathscr{F}$ of $L^{2}(U)$ is a linear isomorphism onto $S^{2}(\Lambda)$. Hence, to see that $\mathscr{F}_{\mathscr{A}}=\left.\mathscr{F}_{\mathscr{F}}\right|_{\mathscr{A}(U)}$ gives a topological linear isomorphism of $\mathscr{A}(U)$ onto $S_{\mathscr{A}}(\mathbb{\Lambda})$, it is sufficient to show that both $\mathscr{F} \mathscr{A}(U) \sqsubset S_{\mathscr{A}}(\mathbb{A})$ and $\mathscr{F}^{-1} S_{\mathscr{A}}(\Lambda) \subset$ $\mathscr{A}(U)$, and that both $\mathscr{F}_{\mathscr{A}}$ and $\mathscr{F}_{\mathscr{S}}{ }^{-1}$ are continuous with respect to the topologies of $\mathscr{A}(U)$ and $S_{\mathscr{A}}(\mathbb{A})$, respectively. The spaces $\mathscr{A}(U)$ and $S_{\mathscr{A}}(\mathbb{\Lambda})$ are endowed with the inductive limit topologies (3.7) and (3.9), respectively. So, to see the continuity of $\mathscr{F}_{\mathscr{A}}$, it is sufficient to prove that for any $\varepsilon>0$ there exists $\varepsilon^{\prime}>0$ such that the restriction of $\mathscr{F}_{\mathscr{F}}$ to the Banach space $\mathbb{O}_{b}\left(V_{\varepsilon}\right)$ gives a bounded linear operator intc the Banach space $S_{-\varepsilon^{\prime}}(\Lambda)$ (cf. [KA, Remarks 2 and 6 in $\$ 5.6$ in Chap. XI]). Since the well-known Weyl dimension formula $d(\lambda)=\operatorname{dim}_{\mathbf{C}} \Gamma(\lambda)$ $=\mathbb{I}_{\alpha \in \Psi}(\lambda+\rho, \alpha) /(\alpha, \alpha)$ implies that $d(\lambda)$ is a polynomial of degree $\Psi$ in $\lambda$, the fact $\mathscr{F} \mathscr{A}(U) \subset S_{\mathscr{A}}(\Lambda)$ and the continuity of $\mathscr{F} \&$ follow from Lemma 3. Next, for $R>0$ we put $n(R)=\#\{\lambda \in \Lambda ;\|\lambda\| \leq R\}$. Then $n(R)$ is bounded from above by a polynomial in $R$. Hence from (2.10) and the Weyl dimension formula we see $\mathscr{F}^{-1} S_{\mathscr{A}}(\Lambda) \subset \mathscr{A}(U)$, the continuity of $\mathscr{F}_{\mathscr{A}}^{-1}$ and (ii).

\section{\$4 Fourier Series of Hyperfunctions on $U$}

Since $U$ is compact, the space $\mathscr{B}(U)$ of all hyperfunctions on $U$ coincides with the dual space $\mathscr{A}(U)^{\prime}$ of $\mathscr{A}(U)$ (cf. [Ma]). Since $\mathscr{A}(U)$ is a DFS space with the inductive limit topology $(3.7), \mathscr{B}(U)$ is an FS space with the following projective limit topology (cf. [Ko]):

$$
\mathscr{B}(U)=\lim \operatorname{proj}_{\varepsilon>0} \mathscr{O}_{b}\left(V_{\varepsilon}\right)^{\prime}=\lim \operatorname{proj}_{k \in \mathbf{Z}_{>0}} \mathscr{O}_{b}\left(V_{1 / k}\right)^{\prime},
$$

where $\mathscr{O}_{b}\left(V_{\varepsilon}\right)^{\prime}$ denotes the dual space of $\mathscr{O}_{b}\left(V_{\varepsilon}\right)$. Note that $\mathscr{B}(U)=\bigcap_{\varepsilon>0} \mathscr{O}_{b}\left(V_{\varepsilon}\right)^{\prime}$ as a set, and for any $\varepsilon>0, \mathscr{O}_{b}\left(V_{\varepsilon}\right)^{\prime}$ is a Banach space with the operator norm

$$
\|f\|_{\varepsilon}^{\prime}=\sup \left\{|(f, F)| ; F \in \mathscr{O}_{b}\left(V_{\varepsilon}\right),\|F\| \leq 1\right\} \quad \text { for } f \in \mathscr{O}_{b}\left(V_{\varepsilon}\right)^{\prime} .
$$

Let $S_{\mathscr{A}}(\Lambda)^{\prime}$ denote the dual space of $S_{\mathscr{A}}(\Lambda)$. Then $S_{\mathscr{A}}(\Lambda)^{\prime}$ is also an FS space with the following projective limit topology: 


$$
S_{\mathscr{A}}(\Lambda)^{\prime}=\lim \operatorname{proj}_{\varepsilon>0} S_{-\varepsilon}(\Lambda)^{\prime}=\lim \operatorname{proj}_{k \in \mathbf{Z}_{>0}} S_{-1 / k}(\Lambda)^{\prime},
$$

where $S_{-\varepsilon}(\Lambda)^{\prime}$ denotes the dual space of $S_{-\varepsilon}(\Lambda)$. We also note that $S_{\mathscr{A}}(\Lambda)^{\prime}=$ $\cap_{\varepsilon>0} S_{-\varepsilon}(\Lambda)^{\prime}$ as a set. We introduce another FS space by putting

$$
S_{\mathscr{B}}(\Lambda)=\lim \operatorname{proj}_{\varepsilon>0} S_{\varepsilon}(\Lambda) \text {. }
$$

Then the equality $S_{\mathscr{B}}(\Lambda)=\cap_{\varepsilon>0} S_{\varepsilon}(\Lambda)$ also holds.

Lemma 4. The FS space $S_{\mathscr{B}}(\Lambda)$ is isomorphic to the FS space $S_{\mathscr{A}}(\Lambda)^{\prime}$ under the following pairing:

$$
(s, S)=\sum_{\lambda \in \Lambda} d(\lambda) \operatorname{Tr}(s(\lambda) S(\lambda)) \quad \text { for }(s, S) \in S_{\mathscr{B}}(\Lambda) \times S_{\mathscr{A}}(\Lambda) .
$$

Proof. For $\eta \in \mathbb{R}$ we put

$$
S_{\eta}^{2}(\Lambda)=\left\{s \in S(\Lambda) ; \sum_{\lambda \in \Lambda} e^{-\eta|| \lambda||} d(\lambda) \operatorname{Tr}(s(\lambda) s(\lambda) *)<\infty\right\} .
$$

Then $S_{\eta}^{2}(\Lambda)$ are Hilbert spaces with the Hermitian inner product

$$
\left(s, s^{\prime}\right)=\sum_{\lambda \in \Lambda} e^{-\eta\|\lambda\|} d(\lambda) \operatorname{Tr}\left(s(\lambda) s^{\prime}(\lambda)^{*}\right) \quad \text { for } s, s^{\prime} \in S_{n}^{2}(\Lambda) .
$$

Furthermore the spaces $S_{\eta}^{2}(\Lambda)$ and $S_{-\eta}^{2}(\Lambda)$ are dual to each other under the pairing

$$
(s, S)=\sum_{\lambda \in \Lambda} d(\lambda) \operatorname{Tr}(s(\lambda) S(\lambda)) \quad \text { for }(s, S) \in S_{\eta}^{2}(\Lambda) \times S_{-\eta}^{2}(\Lambda) .
$$

Let $0<\varepsilon<\varepsilon^{\prime}$. Since $d(\lambda)$ is a polynomial in $\lambda$ and the number $n(R)=\#\{\lambda \in \Lambda$; $\|\lambda\| \leq R\}$ is bounded from above by a polynomial in $R$, we have the injective and continuous sequences:

$$
\begin{aligned}
S_{-\varepsilon^{\prime}}^{2}(\Lambda) & \rightarrow S_{-\varepsilon^{\prime}}(\Lambda) \rightarrow S_{-\varepsilon}^{2}(\Lambda) \rightarrow S_{-\varepsilon}(\Lambda), \\
S_{\varepsilon^{\prime}}(\Lambda) & \leftarrow S_{\varepsilon^{\prime}}^{2}(\Lambda) \leftarrow S_{\varepsilon}(\Lambda) \leftarrow S_{\varepsilon}^{2}(\Lambda) .
\end{aligned}
$$

Hence we have the topological linear isomorphisms:

$$
\begin{aligned}
S_{\mathscr{A}}(\Lambda)^{\prime} & =\lim \operatorname{proj}_{\varepsilon>0} S_{-\varepsilon}(\Lambda)^{\prime}=\lim \operatorname{proj}_{\varepsilon>0} S_{-\varepsilon}^{2}(\Lambda)^{\prime} \\
& =\lim \operatorname{proj}_{\varepsilon>0} S_{\varepsilon}^{2}(\Lambda)=\lim \operatorname{proj}_{\varepsilon>0} S_{\varepsilon}(\Lambda) \\
& =S_{\mathscr{B}}(\Lambda),
\end{aligned}
$$

where the pairing between $S_{\mathscr{A}}(\Lambda)$ and $S_{\mathscr{B}}(\Lambda)$ is given by $(4.5)$.

Now we are going to discuss on the Fourier transformation of $\mathscr{B}(U)$. Since $\Phi(\lambda)(\lambda \in \Lambda)$ are holomorphic representations of $G$, we can take the pairing 
between the elements $f \in \mathscr{B}(U)$ and the functions $\Phi_{i j}\left(\lambda ; u^{-1}\right) \in \mathscr{A}(U)$ :

$$
\mathscr{F}_{i j}(\lambda)=\left(f, \Phi_{j \ell}\left(\lambda ; u^{-1}\right)\right) \quad(\lambda \in \Lambda, 1 \leq i, j \leq d(\lambda)) .
$$

We put $\mathscr{F} f(\lambda)=\left[\mathscr{F}_{i j}(\lambda)\right]_{1 \leq_{i, j} \leq_{d}(\lambda)} \in M(d(\lambda) ; \mathbb{C})$ and $\mathscr{F}_{f}=\left(\mathscr{F}_{f}(\lambda)\right)_{\lambda \in \Lambda} \in S(\Lambda)$, and call the mapping $\mathscr{F}: f \mapsto \mathscr{F} f$ the Fourier transformation of $\mathscr{B}(U)$. Note that the restriction of this Fourier transformation of $\mathscr{B}(U)$ to $L^{2}(U)$ coincides with that of $L^{2}(U)$ defined in $\S 3$ (compare $(4.12)$ with $(3.11)$ ). Now we can state our second assertion.

Theorem 5. (i) The Fourier transformation $\mathscr{F}$ gives a topological linear isomorphism of $\mathscr{B}(U)$ onto $S_{\mathscr{B}}(\Lambda)$.

(ii) The inverse $\mathscr{F}^{-1}$ of the Fourier transformation $\mathscr{F}$ of $\mathscr{B}(U)$ is given by the series

$$
\mathscr{F}^{-1} s=\sum_{\lambda \in \Lambda} d(\lambda) \operatorname{Tr}\left(s(\lambda)^{t} \Phi(\lambda)\right) \text { for } s \in S_{\mathscr{B}}(\Lambda)
$$

converging in the topology of $\mathscr{B}(U)$.

For the proof of this theorem we make some preparations. The set

$$
\left\{d(\lambda)^{1 / 2} \Phi_{i j}\left(\lambda ; u^{-1}\right) ; \lambda \in \Lambda, 1 \leq i, j \leq d(\lambda)\right\}
$$

forms an orthonormal basis of $L^{2}(U)$. Hence we have an expansion for $f \in$ $L^{2}(U)$ :

$$
f=\sum_{\lambda \in \Lambda} d(\lambda) \sum_{1 \leq \iota_{\jmath} \leq d(\lambda)}\left(f, \Phi_{i j}\left(\lambda ; u^{-1}\right)\right) \Phi_{i j}\left(\lambda ; u^{-1}\right) .
$$

We call this expansion the conjugate Fourier series of $f$. We put

$$
\begin{aligned}
\overline{\mathscr{F}}_{i j}(\lambda) & =\left(f, \Phi_{i j}\left(\lambda ; u^{-1}\right)\right) \\
& =\int_{U} f(u) \Phi_{j \imath}(\lambda ; u) d u \quad(1 \leq i, j \leq d(\lambda)),
\end{aligned}
$$

and $\overline{\mathscr{F}} f(\lambda)=\left[\overline{\mathscr{F}} f_{i j}(\lambda)\right]_{1 \leq_{i j} \leq d(\lambda)} \in M(d(\lambda) ; \mathbf{C})$ for $f \in L^{2}(U)$ and $\lambda \in \Lambda$. We also put $\overline{\mathscr{F}} f=(\overline{\mathscr{F}} f(\lambda))_{\lambda \in \Lambda} \in S^{2}(\Lambda)$, and call the mapping $\overline{\mathscr{F}}: f \mapsto \mathscr{F} f$ the conjugate Fourier transformation of $L^{2}(U)$. The inverse $\overline{\mathscr{F}}^{-1}$ of the mapping $\overline{\mathscr{F}}$ is given by

$$
\overline{\mathscr{F}}^{-1} s=\sum_{\lambda \in \Lambda} d(\lambda) \operatorname{Tr}\left(s(\lambda)^{t} \Phi\left(\lambda ; u^{-1}\right)\right) \text { for } s \in S^{2}(\Lambda) .
$$

As for the restriction of the conjugate Fourier transformation to $\mathscr{A}(U)$ we also have the following theorem.

Theorem $2^{\prime}$. (i) The conjugate Fourier transformation $\overline{\mathscr{F}}$ gives a topological 
linear isomorphism of $\mathscr{A}(U)$ onto $S_{\mathscr{A}}(\Lambda)$.

(ii) For $s \in S_{\mathscr{A}}(\Lambda)$, the conjugate Fourier series (4.17) converges in the topology of $\mathscr{A}(U)$.

Proof. Recalling the proof of Theorem 2, we see that the proof of this theorem is reduced to showing the following two inequalities:

$$
\left|\overline{\mathscr{F}}_{i j}(\lambda)\right| \leq\|f\|_{\varepsilon} e^{-\varepsilon\|\lambda\|} \quad\left(1 \leq_{i, j} \leq_{d}(\lambda)\right)
$$

for all $\lambda \in \Lambda$, all $f \in \mathscr{O}_{b}\left(V_{\varepsilon}\right)$ and all $\varepsilon>0$, and

$$
\left|\Phi_{i j}\left(\lambda ; g^{-1}\right)\right| \leq e^{\|\lambda\|\|g\|} \quad(1 \leq i, j \leq d(\lambda))
$$

for all $\lambda \in \Lambda$ and all $g \in G$. The proof of (4.18) is similar to that of (3.10), and the inequality (4.19) follows from (2.10) and (2.8).

Proof of Theorem 5. (i) The fact $\mathscr{F}_{\mathscr{F}} \mathscr{B}(U) \subset S_{\mathscr{B}}(\Lambda)$ follows from (4.19) and the Weyl dimension formula. We have seen in Theorem $2^{\prime}$ that the series (4.17) for $S \in S_{\mathscr{A}}(\mathbb{A})$ converges in the topology of $\mathscr{A}(U)$. Then by (4.5) we have

$$
\begin{aligned}
\left(f, \overline{\mathscr{F}}^{-1} S\right) & =\left(f, \sum_{\lambda \in \Lambda} d(\lambda) \operatorname{Tr}\left(S(\lambda)^{t} \Phi\left(\lambda ; u^{-1}\right)\right)\right) \\
& =\sum_{\lambda \in \Lambda} d(\lambda) \operatorname{Tr}(S(\lambda) \mathscr{F} f(\lambda)) \\
& =\left(\mathscr{F}_{f} f, S\right)
\end{aligned}
$$

for all $f \in \mathscr{B}(U)=\bigcap_{\varepsilon>0} \mathscr{O}_{b}\left(V_{\varepsilon}\right)^{\prime}$. This shows that the Fourier transformation $\mathscr{F}_{\mathscr{B}}$ of $\mathscr{B}(U)$ coincides with the dual operator $\left(\overline{\mathscr{F}}_{\mathscr{A}}{ }^{-1}\right)^{d}$ of $\overline{\mathscr{F}}_{\mathscr{A}}{ }^{-1}$. Hence again from Theorem $2^{\prime}$ and Lemma 4 we see that the operator $\mathscr{F}_{\mathscr{B}}$ gives a topological linear isomorphism of $\mathscr{B}(U)$ onto $S_{\mathscr{B}}(\Lambda)$.

(ii) Let $s \in S_{\mathscr{B}}(\mathbb{\Lambda})=\cap_{\varepsilon>0} S_{\varepsilon}(\mathbb{\Lambda})$. By virtue of (4.18) we see that the series (4.13) for $s$ converges in the operator norm \|\|$_{\varepsilon}^{\prime}$ for all $\varepsilon>0$. This means that it converges in the topology of $\mathscr{B}(U)$ (cf. [KA, Remark 1 in $\S 5.2$ in Chap. XI]). Then again by $(4.5)$ we have

$$
\begin{aligned}
\left(\sum_{\lambda \in \Lambda} d(\lambda) \operatorname{Tr}\left(s(\lambda)^{t} \Phi(\lambda ; u), F\right)\right) & =\sum_{\lambda \in \Lambda} d(\lambda) \operatorname{Tr}(s(\lambda) \overline{\mathscr{F}} F(\lambda)) \\
& =(s, \overline{\mathscr{F}} F)
\end{aligned}
$$

for all $s \in S_{\mathscr{B}}(\Lambda)$ and all $F \in \mathscr{A}(U)$. This shows that the series (4.13) for $s \in$ $S_{\mathscr{D}}(\Lambda)$ coincides with $\left(\overline{\mathscr{F}}_{\mathscr{A}}\right)^{d} s$, where $\left(\overline{\mathscr{F}}_{\mathscr{A}}\right)^{d}$ denotes the dual operator of $\overline{\mathscr{F}}_{\mathscr{A}}$. On the other hand, from the proof of $(\mathrm{i})$ we see $\left(\overline{\mathscr{F}}_{\mathscr{A}}\right)^{d}=\mathscr{F}_{\mathscr{B}}{ }^{-1}$. Thus we see that the series (4.13) for $s \in S_{\mathscr{B}}(\Lambda)$ coincides with $\mathscr{F}_{\mathscr{B}}{ }^{-1} s$. 


\section{Acknowledgement}

The author is grateful to the referee and Prof. Oshima, who taught the author that we should take the norm of the elements $g \in G$ via the Cartan decomposition not via the Iwasawa decomposition even in our case and that the estimate (2.10) is generally known. By these advice we could make the paper more concise and the results sharper.

\section{References}

[A] Arthur. J. G., Harmonic analy'sis of tempered distributions on semisimple Lie groups of real rank one. Ph. D. Thesis, Yale Univ., 1970.

[B1] Ban. E. P. van den, Asymptotic expansions and integral formulas for eigenfunctions on a semısımple Lie group. Doctor thesis. Rijksuniversiteit Utrecht, 1982.

[B2] _. Asymptotic behaviour of Eisenstein integrals, Bull. (New Series) AMS, 9 (1983), 311-314.

[E] Eguchi, M., Asymptotic exansions of Eisenstein integrals and Fourier transform on symmetric spaces. J. Funct. Anal., 34 (1979), 167-216.

[FM] Fujıi, K. and Morimoto, M., Gevrey classes on compact real analytic Riemannian manifolds, Tokyo J. Math., 18 (1995), 341-355.

[HC1] Harısh-Chandra, Representations of semisimple Lie groups II. Trans. Amer. Math. Soc., 76 (1954), 26-65.

[HC2] _. Spherical functions on a semisimple Lie group I, Amer. J. Math., 80 (1958), 241-310.

[HC3] _. idem II; Amer. Math. J. Math., 80 (1958), 553-613.

[HC4] . Harmonic analysis on real reductive groups III, Ann. of Math., 104 (1976), 117-201.

[He] Helgason. S., Differential Geometry, Lie Groups, and Symmetric Spaces, Academic Press, New York, 1978.

[HMO] Hashizume, M., Minemura, K. and Okamoto, K., Harmonic functions on hermitian hyperbolic spaces, Hiroshima Math. J., 3 (1973), 81-108.

[Hu] Hua, L.-K., Harmonic Analysis of Functions of Several Complex Variables in Classical Domains, Moscow, 1959 (in Russian): Transl. Math. Monographs 6, AMS. Providence, Rhode Island, 1963.

[KA] Kantorovich, L. V. and Akilov, G. P., Functional Analysis in Normed Spaces, Fizmatgiz, Moscow, 1959 (in Russian); English translation from Pergamon Press, Oxford, 1964.

[Kn] Knapp. A. W., Representation Theory of Semisimple Lie Groups, Princeton Univ. Press, Princeton, 1986.

[Ko] Komatsu. H., Projective and injective limits of weakly compact sequenses of locally convex spaces, J. Math. Soc. Japan, 19 (1967), 367-383.

[Ma] Martineau, A., Les hyperfonction de M. Sato, Sém. Bourbaki, 13 (1961), No. 214.

[Mi] Minakshisundaram, S., A generalization of Epstein zeta functions, Canadian J. Math., 1 (1949), 320-327.

[Mo1] Morimoto, M., Analytic functionals on the Lie sphere, Tokyo J. Math., 3 (1980), 1-35.

[Mo2] , Analytic functionals on the sphere and their Fourier-Borel transformations, 
Complex Analysis, Banach Center Publ., 11, 223-250, PWN-Polish Scientific Publishers. Warsaw, 1983.

[OSW1] Oshima, T., Saburi, Y. and Wakayama, M., A note on Ehrenpreis' fundamental principle on a symmetric space, in Algebraic Analysis, Vol. II, 681-697, edited by Kashiwara. M. and Kawai, T., Academic Press, 1988.

[OSW2] __ Paley-Wiener theorems on a symmetric space and their application. Diff. Geom. Appl., 1 (1991), 247-278.

[Sa] Sato, M., Theory of hyperfunctions, Sugaku, 10 (1958), 1-27 (in Japanese).

[Su] Sugiura, M., Fourier series of smooth functions on compact Lie groups, Osaka J. Math., 8 (1971), 33-47.

[TV] Trombi, P. C. and Varadarajan, V. S., Spherical transforms on semisimple Lie groups, Ann. of Math., 94 (1971), 246-303.

[W] Wallach, N. R., Real Reductive Groups I, Academic Press, San Diego, 1988. 